\title{
Innerfamiliale Beziehungen und Bildungserfolg
}

\section{$\boldsymbol{Z f E}$}

\author{
Monja Schmitt
}

Zusammenfassung: Coleman formuliert die Annahme, dass Sozialkapital als Ressource in den sozialen Beziehungen zwischen den Familienmitgliedern liegt und sich unabhängig anderer Ressourcen auf die schulische Performanz der Kinder auswirkt. In Anbetracht der frühen Aufteilung in die verschiedenen Sekundarschulzweige des deutschen Bildungssystems wird - je näher der Übertritt rückt - nicht nur untersucht, inwiefern sich das familiale Miteinander zu einem bestimmten Zeitpunkt, sondern auch wie sich eine beobachtete Veränderung der Eltern-KindBeziehung auf den Bildungserfolg auswirkt. National und auch international liegen nur wenige längsschnittliche Ergebnisse innerhalb dieses Forschungsfeldes vor.

Multinomiale logistische Regressionen zeigen, dass sowohl Strukturmerkmale als auch Prozessmerkmale einer engen Eltern-Kind-Beziehung einen signifikant eigenständigen Einfluss auf schulischen Erfolg haben. Erklärkraft haben dabei nicht nur Indikatoren schulischer Interaktion, sondern auch solche, die den familialen Alltag abbilden. Veränderungen in der Eltern-Kind-Beziehung spielen dann eine Rolle, wenn es sich um eine vom Kind wahrgenommen Veränderung einer schulbezogenen Interaktion handelt.

Schlüsselwörter: Soziale Beziehungen · Sozialkapital · Längsschnittstudien · Bildungserfolg · Eltern-Kind-Beziehung

\section{Intrafamiliar relations and educational success}

\begin{abstract}
According to Coleman, social capital lies in the social relations between parents and children and constitutes a child's social background in addition to the family's human capital and economic resources. In this perspective, Coleman emphasizes the importance of social relations as an independent third factor for the acquirement of human capital. Considering the early selection into the different types of secondary education in the German educational system after fourth grade, not only effects of social relations at this decisive point in time are investigated, but also changes across time towards this transition. Therefore, using longitudinal data is of special advantage in this field of research, which is dominated by cross-sectional analyses.

Applying multinomial logistic regression it is shown that structural aspects as well as process-related aspects of the parent-child-relation have a significant effect on academic achievement. Under this perspective indicators for school-related parent-child interaction are as important as
\end{abstract}

Online publiziert: 14.01 .2010

(C) Die Autoren 2010. Dieser Artikel ist auf Springerlink.com mit Open Access verfügbar.

M. Schmitt, M.A. ( $\bowtie)$

Forschergruppe ,Bildungsprozesse, Kompetenzentwicklung und Selektionsentscheidungen im Vor- und Grundschulalter' (BiKS), Otto-Friedrich-Universität Bamberg

Jäckstraße 3, 96052 Bamberg, Deutschland

E-Mail: monja.schmitt@uni-bamberg.de 
everyday interaction. Additionally, positive change of school-related parent-child interactions affects academic achievement.

Keywords: Academic achievement · Longitudinal studies · Parent-child-relation ·

Social capital $\cdot$ Social relations

\section{Einleitung}

Die Bildungskarrieren in Deutschland werden vor allem durch die Weichenstellung am Ende der Grundschulzeit entscheidend geprägt. Der Übergang in den Sekundarschulbereich kann dementsprechend als „sensible Phase“ verstanden werden (vgl. Blossfeld 1988). Stark beeinflusst wird der Übergang sowohl durch kindliche Kompetenzen und Schulnoten (vgl. z. B. Baumert et al. 2001; Prenzel et al. 2004) als auch durch eine unterschiedliche Ausstattung an Ressourcen des Elternhauses und den damit verbundenen unterschiedlichen Bildungsentscheidungen (vgl. z.B. Becker 2000). Die Bildungsforschung der letzten Jahre zeigte, dass das deutsche Bildungssystem nach wie vor kein Ort sozial gleicher Bildungschancen ist (vgl. z. B. Blossfeld 1993; Müller u. Haun 1994; Schimpl-Neimanns 2000; Becker 2004). Gezeigt werden konnten Effekte für das familiale Humankapital, die sozio-ökonomische Position (vgl. z. B. Schimpl-Neimanns 2000) und den Migrationsstatus (vgl. z. B. Büchel u. Wagner 1996; Kristen 2002) sowie Effekte der regionalen Herkunft der Familie (vgl. z. B. Henz u. Maas 1995; Ehmke et al. 2005), der Lehrerempfehlung (vgl. z. B. Bos et al. 2004; Ditton et al. 2005), des Klassenkontextes (vgl. z. B. Kristen 2002) und der institutionellen Umwelt auf die Wahl einer weiterführenden Schule (vgl. z. B. von Below 2002).

Neben den genannten Faktoren stellt die Ressource Sozialkapital eine weitere für den Bildungserfolg wichtige Determinante dar. Kinder aus Familien mit hohem Sozialkapital erzielen höhere Werte in Kompetenztestungen, bessere Noten und verlassen die Schule seltener vorzeitig (vgl. z. B. Israel et al. 2001; Stecher 2001; Teachman et al. 1996). Dabei können verschiedene Definitionen von Sozialkapital herangezogen werden, die auf den Arbeiten von Bourdieu (1983) und Coleman (1988) basieren. Während in den Arbeiten Bourdieus ökonomisches, kulturelles und soziales Kapital eng miteinander verbunden sind, geht Coleman von einer unabhängigen Funktion des Sozialkapitals aus. Sozialkapital als Ressource eines Kollektivs nach Bourdieu wird dem einzelnen über die mehr oder weniger institutionalisierten Beziehungen zwischen den Mitgliedern dieses Kollektivs zugänglich. Dabei betont Bourdieu besonders die Instrumentalisierung von Sozialkapital durch die herrschende Klasse als Ressource zur Machterhaltung. In Abgrenzung dazu sieht Coleman Sozialkapital als in der Struktur der Beziehungen liegende Ressource, die zu bestimmten Handlungen befähigt bzw. diese erleichtert. Er betont insbesondere die Verantwortung der Familie, über intergenerationale Geschlossenheit Kindern bessere Lebenschancen zu ermöglichen.

Den theoretischen Annahmen und empirischen Befunden Colemans folgend, soll unter Hinzuziehung von längsschnittlichen Daten der Forschergruppe BiKS-8-12 ${ }^{1}$ geklärt werden, ob sich die Art der Eltern-Kind-Beziehung auf den Bildungserfolg vor dem Übergang von der Grundschule auf eine weiterführende Schule auswirkt. Den Vorteilen der 
Nutzung eines längsschnittlichen Designs soll dabei besondere Aufmerksamkeit gewidmet werden.

Zunächst werden der theoretische Hintergrund und der Forschungsstand erläutert, gefolgt von der Datenbasis, den zentralen Variablen und der methodischen Vorgehensweise. Im Anschluss daran wird mit Hilfe multinomialer logistischer Regressionsanalysen untersucht, ob das Verhältnis zwischen Eltern und Kind einen eigenständigen Beitrag zur Erklärung von Schulleistungen liefert. Abschließend werden die Befunde interpretiert und diskutiert.

\section{Theoretischer Hintergrund, Forschungstand und Fragestellung}

\subsection{Theoretischer Hintergrund}

Soziale Beziehungen zwischen Akteuren bergen nach Coleman (1988, 1990) Sozialkapital als Ressource. Coleman unterscheidet dabei bestimmte Merkmale sozialer Beziehungen, die diese zu einer wertvollen Handlungsressource werden lassen. Betont wird Sozialkapital in Form von Vertrauen in die Reziprozität sozialer Beziehungen, also der Überzeugung, dass die soziale Beziehung gegenseitig ist und dadurch gegenseitige Verpflichtungen und Erwartungen bestehen. Darüber hinaus fungieren soziale Beziehungen als Informationskanäle, sie schaffen Normen und Sanktionen, die neben dem Vertrauen in die Gegenseitigkeit der Beziehung wichtiges Handlungspotenzial besitzen. Coleman erläutert weiterhin, dass folgende Strukturen sozialer Beziehungen der Entstehung von Sozialkapital zuträglich sind: Die Geschlossenheit der sozialen Beziehung und die geeignete soziale Einbettung dieser Beziehung. Er verweist über diese allgemeinen Überlegungen hinaus aber insbesondere auf die Relevanz sozialer Beziehungen für den Erwerb von Humankapital. Dabei unterscheidet er zwischen sozialem Kapital innerhalb der Familie, also der Eltern-Kind-Beziehung und sozialem Kapital innerhalb der Gemeinschaft (außerhalb der Familie). Sozialkapital innerhalb der Familie ist nach Coleman neben den ökonomischen Ressourcen und dem Humankapital die dritte Komponente, die den familialen Hintergrund eines Kindes abbildet. Während die finanziellen Ressourcen einer Familie für die Möglichkeit stehen, in materieller Hinsicht eine lernförderliche Umgebung (eigener Schreibtisch, zusätzliche Lernmittel, Bücher u.ä.) zu schaffen, steht das Humankapital für eine kognitiv anregende Atmosphäre innerhalb der Familie. Die Rolle des Eltern-Kind-Verhältnisses für den Erwerb von Humankapital lässt sich desgleichen nicht ähnlich deutlich fassen oder abgrenzen. Der zeitliche Umfang, den Eltern ihren Kindern zuteil werden lassen, ist ebenso wichtig wie elterliche Erwartungen, der elterliche Unterstützungswille, elterliche Aufmerksamkeit oder das Bemühen der Eltern um ihre Kinder. Zusammenfassend unterscheidet Coleman also innerhalb der Familie nicht mehr die verschiedenen Formen von Sozialkapital, sondern betont letztlich die Enge und Stärke der Eltern-Kind-Beziehung. Er verweist dabei auf Beispiele einer engen ElternKind-Beziehung, welche sich zuträglich auf den Lernerfolg eines Kindes ausgewirkt hatten - ohne dass die Familien über hohes Humankapital oder eine gehobene sozioökonomische Position verfügt hätten. Coleman zufolge ist Sozialkapital also eine Ressource, die sich über ihre eigenständige Funktion definiert und nicht über ihren engen 
Zusammenhang zwischen ökonomischen Kapital und Humankapital. Smith et al. (1995) erweitern den Begriff des Sozialkapitals, indem sie ihn in eine strukturelle und prozessuale Komponente differenzieren. Indikatoren einer bestimmten Struktur einer sozialen Beziehung bilden sowohl die Möglichkeiten zu interagieren als auch die Häufigkeit und Dauer einer sozialen Interaktion ab. Prozessmerkmale einer sozialen Beziehung repräsentieren im Gegensatz zu den strukturellen Komponenten, die den quantitativen Aspekt der Eltern-Kind-Interaktion betonen, die Qualität des Eltern-Kind-Verhältnisses. In Anlehnung an Coleman sind die Anzahl der Betreuungspersonen oder die Zahl der Geschwister Strukturmerkmale einer sozialen Beziehung, welche die Quantität des Eltern-Kind-Verhältnisses abbilden. Prozessmerkmale (und somit der qualitative Aspekt der Eltern-KindBeziehung) beinhalten u. a. die Unterstützung bei den Hausaufgaben, Diskussionen über schulische Angelegenheiten oder Bildungsaspirationen.

\subsection{Forschungsstand und Fragestellung}

In empirischen Analysen bestätigt Coleman (1988) seine theoretischen Überlegungen. Er kann signifikante Zusammenhänge einer engen Eltern-Kind-Beziehung und der Schulabbrecherquote nachweisen und findet dabei sowohl Effekte familialer Strukturmerkmale wie physische An- bzw. Abwesenheit der Eltern und die Zahl der Geschwister als auch Effekte mütterlicher Bildungserwartungen als Prozessmerkmal. Die Anzahl der Gespräche über persönliche Erfahrungen zeigt in diesen Analysen keine signifikanten Zusammenhänge mit der Schulabbrecherquote. Auch Untersuchungen anderer Autoren finden Zusammenhänge struktureller und prozessualer Merkmale des Sozialkapitals mit Bildungserfolg im weiteren Sinne. In Konsens mit Coleman belegen zahlreiche Analysen (vgl. z. B. Israel et al. 2001; Smith et al. 1992, 1995), dass Kinder aus alleinerziehenden Haushalten und mit vielen Geschwistern signifikant schlechtere Noten erzielen und häufiger die Schule abbrechen. Auch Stecher (2001) verweist auf die Bedeutung einer intakten Familienstruktur für den Bildungserfolg (Notendurchschnitt) von Jugendlichen. Smith et al. (1992) ergänzen eine weitere Strukturvariable und finden, dass Kinder, deren Mütter vor ihrer Einschulung berufstätig waren, häufiger die Schule abbrechen.

Ebenfalls in Übereinstimmung mit Coleman zeigen Israel et al. (2001), dass sich eine hohe Bildungsaspiration mindestens eines Elternteils signifikant positiv auf das Erzielen von hohen Kompetenztestwerten, guten Noten und das Verbleiben an einer Schule auswirken (vgl. dazu auch Smith et al. 1992; Stecher 2001). Darüber hinaus können die Autoren diese Zusammenhänge auch für das Diskutieren von schulischen Belangen nachweisen (vgl. auch Ho Siu-Chu u. Willms 1996; Teachman et al. 1996), während die Anzahl der Gespräche über persönliche Angelegenheiten (vgl. auch Coleman 1988; Smith et al. 1992) keine statistisch bedeutsamen Effekte auf das Abbrechen der Schule hat. Dohle u. Wessel (1997) finden desgleichen positive Effekte einer schulunabhängigen, intensiven Eltern-Kind-Interaktion - gemessen an gemeinsamen Aktivitäten und einem guten Vertrauensverhältnis auf schulische Leistungen. Ho Sui-Chu u. Willms (1996) zeigen weiterhin, dass die Häufigkeit des Beschränkens der vor dem Fernseher verbrachten Zeit, das Beschränken der Ausgehzeit, das Überwachen von Hausaufgaben und die Anwesenheit eines Elternteils nach der Schule signifikant positiv auf das Erzielen hoher Kompetenztestwerte wirken. Alle vier Indikatoren gingen als ein Faktorwert in die Analysen ein. 
Während die Häufigkeit des Beschränkens der vor dem Fernseher verbrachten Zeit auch als Einzelindikator in die erwartete Richtung zeigt (vgl. Israel et al. 2001; Israel u. Beaulieu 2002), ergeben sich für die Unterstützung bei den Hausaufgaben andere Effekte. Jungbauer-Gans (2004) zeigt, dass die familiale Unterstützung bei den Hausaufgaben signifikant negativ mit dem Erzielen von Kompetenztestwerten zusammenhängt (vgl. auch Israel et al. 2001; Israel u. Beaulieu 2002). Möglicherweise kommt bei den Kindern durch zu häufige Hausaufgabenkontrolle das Gefühl von übermäßigem Druck auf oder die Familie verhindert durch zu viel Unterstützung das tatsächliche Sicheinstellen eines Lernerfolgs. Denkbar wäre auch, dass gerade Kinder mit schlechten schulischen Leistungen von den Eltern besondere Unterstützung benötigen. Klarheit über die Richtung dieses Effektes können längsschnittliche Analysen schaffen. Zusammenfassend stehen genannte Indikatoren für die Qualität der Eltern-Kind-Interaktion bzw. der Familienbeziehung (vgl. auch Stecher 2001). Es werden dabei nicht nur solche Indikatoren einer Eltern-Kind-Beziehung berücksichtigt, die die Interaktion über schulische Angelegenheiten abbilden, sondern auch solche eines alltäglichen Umgangs miteinander.

Gemeinsam ist allen aufgeführten Untersuchungen, dass zumeist Angaben der Eltern operationalisiert wurden, d.h. die Sicht der Kinder nicht berücksichtigt werden konnte. Lediglich Jungbauer-Gans (2004) und Stecher (2001) haben die Sichtweise der Kinder mit in die Analysen aufgenommen. Weiterhin gemeinsam ist den angeführten Analysen, dass Auswirkungen von Sozialkapital auf Bildungserfolg stets für Kinder und Jugendliche in einem Alter untersucht wurde, welches - übertragen auf das deutsche Bildungssystem - erst nach dem Übergang in den Sekundarbereich liegt.

Signifikante Effekte sozialer Beziehungen innerhalb der Familie können auch unter Berücksichtigung familialer Ressourcen nachgewiesen werden und haben damit einen eigenständigen Ressourceneffekt auf den Bildungserfolg. Gemäß der theoretischen Überlegungen Colemans finden familiale Ressourcen über Bildungsabschlüsse der Eltern und finanzielle Ressourcen der Familie (vgl. z. B. Teachman et al. 1996) Eingang in die empirischen Analysen. Vorliegende Untersuchungen bilden sowohl die familialen Ressourcen als auch die sozio-ökonomische Position der Familie ab (vgl. z. B. Ho Sui-Chu u. Willms 1996).

Bildungserfolg als abhängige Variable wird in den dargestellten Untersuchungen unterschiedlich operationalisiert. Das Erzielen von Kompetenztestwerten und Noten wird ebenso verwendet wie das Beenden der besuchten Schule (für einen Vergleich z. B. Israel et al. 2001). Angepasst an das deutsche Bildungssystem wäre hier auch der für die Bildungskarriere zentrale Übergang in den Sekundarbereich I anzuführen (vgl. z. B. Stecher 2001).

Gemäß den angeführten theoretischen Überlegungen und den empirischen Ergebnissen wird Sozialkapital in Form der Eltern-Kind-Beziehung also zu einem Faktor, der Bildungserfolg positiv beeinflusst. Qualitative Aspekte des innerfamilialen Verhältnisses werden dabei ebenso bedeutsam wie strukturelle Merkmale; die signifikant eigenständige Wirkung sozialer Beziehungen auf den Bildungserfolg konnte in zahlreichen Studien nachgewiesen werden. Ziel folgender Untersuchung soll es zunächst sein, diese Ergebnisse zu replizieren. Erweiternd soll die, bisher in den seltensten Fällen berücksichtigte, Sichtweise der Kinder einbezogen sowie - über die Nutzung eines längsschnittlichen Designs - die Wirkweise der verwendeten Indikatoren festgelegt werden. 
In den ersten empirischen Analysen wird daher versucht, folgende Fragen zu klären, die sich auf einen früheren Zeitpunkt der Bildungskarriere beziehen: die Phase vor dem Übergang von der Primar- auf die Sekundarstufe I: Kann ein signifikanter Zusammenhang zwischen einer intensiven Eltern-Kind-Beziehung und schulischem Erfolg gezeigt werden? Bleibt dieser Zusammenhang auch unter Berücksichtigung der familialen Ressourcen bestehen? Haben sowohl strukturelle als auch prozessuale Merkmale der ElternKind-Beziehung einen Effekt auf schulischen Erfolg? Sind sowohl das schulische als auch das alltägliche Eltern-Kind Verhältnis geeignete Prädiktoren schulischen Erfolges? Lassen sich sowohl für Indikatoren aus Kindsicht als auch aus Elternsicht Effekte zeigen?

Im zweiten empirischen Teil soll - bezogen auf eine Besonderheit des deutschen Bildungssystems - ein weiterer bisher noch wenig untersuchter Aspekt thematisiert werden. Die angeführten Studien untersuchen die Auswirkungen innerfamilialen sozialen Kapitals, zumeist ohne dabei den Zeitpunkt dieser Interaktion speziell zu berücksichtigen oder zu akzentuieren. Innerhalb des deutschen Bildungssystems wird bereits nach der vierten Grundschulklasse eine Entscheidung über die folgende Bildungskarriere verlangt, was diesen Zeitpunkt zu einer zentralen Schnittstelle in der Bildungskarriere eines Kindes werden lässt. Die im Lebenslauf frühe Aufteilung in die verschiedenen Zweige der Sekundarstufe wird folglich zu einer „sensiblen Phase“ (vgl. u. a. Blossfeld 1988). Verstärkend kommt hinzu, dass ein Aufstieg zwischen den verschiedenen Bildungszweigen nur selten erfolgt (vgl. z. B. Hillmert u. Jacob 2005). Je näher also diese Phase der schulischen Laufbahn und damit die Entscheidung über den zu vollziehenden Übergang kommt, desto mehr rückt dieses Thema bei Lehrern, Kindern und Eltern in den Vordergrund (vgl. z. B. Büchner u. Koch 2001; Pohlmann 2008). Schulbezogene als auch alltägliche familiale Prozesse - die Qualität des familialen Miteinanders - sind dementsprechend zu genau diesem Zeitpunkt besonders wichtig und sollten sich darüber hinaus verändern, je näher der Bildungsübergang rückt.

Ausgehend von diesen Überlegungen stehen deswegen im zweiten Teil der empirischen Analysen folgenden Fragen im Mittelpunkt: Gibt es Veränderungen in der Eltern-KindInteraktion, je näher der Übergang rückt? Wirken sich Veränderungen in der Eltern-KindBeziehung auf den schulischen Erfolg aus? Sind nur solche Veränderungen relevant, die den schulbezogenen Umgang der Eltern mit den Kindern abbilden oder auch Veränderungen in der alltäglichen Interaktion?

\section{Empirischer Teil}

\subsection{Forschungsdesign und Stichprobe}

Zur Analyse der aufgeworfenen Forschungsfragen werden Daten der interdisziplinären Forschergruppe BiKS „Bildungsprozesse, Kompetenzentwicklung und Selektionsentscheidungen im Vor- und Grundschulalter" verwendet. Das an der Otto-Friedrich-Universität Bamberg angesiedelte Projekt besteht aus einem Team von Wissenschaftlerinnen und Wissenschaftlern der Soziologie, Pädagogik und Psychologie und hat sich zum Ziel gesetzt, Prozesse des Kompetenzerwerbs und der Entscheidungsbildung im Vor- und Grundschulalter genauer zu untersuchen. Im Zentrum des Forschungsprojektes stehen 
zwei Längsschnittstudien: BiKS-3-8 und BiKS-8-12. Relevant für die vorliegende empirische Arbeit sind Daten des Längsschnitts BiKS-8-12, welcher die Herausbildung von Entscheidungen beim Übergang von der Grundschule in den Sekundarbereich in Abhängigkeit vom Kompetenzstand der Kinder sowie von strukturellen und einstellungsbezogenen Einflüssen untersucht. Hierzu werden die Entwicklungen der Kinder von der dritten bis zur siebten Klasse verfolgt.

Bei der Stichprobenziehung der beiden Längsschnittstudien wurden zunächst mit Bayern und Hessen zwei Bundesländer ausgewählt, die sich in ihren institutionellen Übergangsregelungen unterscheiden. Im Anschluss daran wurden städtische und ländliche Regionen nach einem disproportional geschichteten Stichprobenplan gewählt. Die Stichprobe der Grundschulen von BiKS-8-12 wurde nach einer disproportional geschichteten Zufallsstichprobe von Kindergärten und einer daran gekoppelten Auswahl von Grundschulen bestimmt (vgl. Kurz et al. 2007). Realisiert werden konnte im Längsschnitt BiKS-8-12 (erste Welle März 2006) eine Fallzahl von 2.395 Schülerinnen und Schüler aus 155 Schulklassen, in denen in etwa halbjährlichem Abstand umfangreiche Erhebungen durchgeführt werden (Maurice et al. 2007). Bei den Erhebungen kommen neben standardisierten Kompetenzmessungen standardisierte Befragungen von Eltern, Lehrkräften und Kindern zum Einsatz. Zudem finden qualitative Zusatzerhebungen an einer Teilstichprobe mit Eltern und Lehrkräften statt. Eine Auswahl an Stichprobenmerkmalen des Längsschnitts BiKS-8-12 ist in Tab. 1 dargestellt.

Die Kinder sind bei Eintritt in den Schullängsschnitt durchschnittlich knapp neun Jahre alt. Die Gesamtstichprobe von 2.395 Kindern besteht aus etwas mehr Jungen als Mädchen, die insgesamt 155 Schulklassen (designbedingt) zu 65\% in Bayern und 35\% in Hessen besuchen. In der BiKS-Stichprobe befinden sich knapp 25\% Familien, bei denen mindestens ein Elternteil nicht in Deutschland geboren ist. In etwa 25\% der Familien liegt höchstens der qualifizierende Hauptschulabschluss als höchster allgemeinbildender Schulabschluss vor. Ein Drittel der Familien hat höchstens die Mittlere Reife als höchsten familialen Bildungsabschluss und in fast 43\% der Familien ist mindestens die Fachhochschulreife im Haushalt vorhanden. 38\% Prozent der befragten Haushalte erzielen ISEI-Werte (International SocioEconomic Index of Occupational Status - vgl. Ganzeboom et al. 1992) im unteren Viertel, während 36\% der Haushalte ISEI-Werte im oberen Viertel erreichen.

Tab. 1: Ausgewählte Stichprobenmerkmale des Längsschnitts BiKS-8-12

\begin{tabular}{ll}
\hline $\begin{array}{l}\text { Stichprobenumfang } \\
\text { Alter des Kindes bei Eintritt in die }\end{array}$ & $\mathrm{N}=2.395$ aus 155 Schulklassen \\
$\begin{array}{l}\text { Studie (März 2006) } \\
\text { Geschlecht des Kindes }\end{array}$ & \\
Wohnort des Kindes & $52,2 \%$ männlich; $47,8 \%$ weiblich \\
ISEI & $35,0 \%$ Hessen; $65,0 \%$ Bayern \\
& AM 50,48; Min $=16 ;$ Max $=90 ; \mathrm{SD}=16,41$ \\
Höchster Bildungsabschluss in der & Perzentile $25=38 \% ; 50=51 \% ; 75=64 \%$ \\
Familie & $24,0 \%$ max. Qualifizierender Hauptschulabschluss \\
& $33,1 \%$ max. Mittlerer Reife \\
Migrationshintergrund & $42,9 \%$ mind. Fachhochschulreife \\
& $76,2 \%$ ohne Migrationshintergrund \\
& $23,8 \%$ mind. ein Elternteil nicht in Deutschland geboren \\
\hline
\end{tabular}




\subsection{Instrumente und Variablen zur Operationalisierung sozialer Beziehungen}

Bis Ende 2008 wurden im Rahmen des Längsschnitts BiKS-8-12 vier Messzeitpunkte realisiert. Die Auswertungen des vorliegenden Beitrages beziehen sich auf Angaben aus Welle eins (März 2006, Mitte dritte Klasse) und Welle zwei (September 2006, Anfang vierte Klasse). ${ }^{2}$ In die Analysen einbezogen werden also Kinder zu einem frühen Zeitpunkt der Bildungskarriere vor dem Bildungsübergang. Genutzt werden zum einen die Ergebnisse der telefonischen Befragungen der Eltern, in der neben den für die Fragestellung relevanten Angaben u. a. auch Angaben zum Bildungshintergrund und der sozio-ökonomischen Position erfragt wurden sowie Ergebnisse der schriftlichen Schülerbefragungen im Klassenkontext. Zum anderen werden Lehrerangaben zu Noten verwendet. Ein Vorzug der Fülle an Indikatoren ist, dass neben strukturellen Merkmalen die prozessualen Merkmale nicht nur als Indikatoren eines schulbezogenen Umgangs der Eltern mit dem Kind erfragt wurden, sondern auch Indikatoren der alltäglichen Eltern-Kind-Beziehung berücksichtigt werden können. Ein weiterer Vorteil der verwendeten Daten liegt darin, dass sowohl die Perspektive der Eltern als auch die Wahrnehmung der Kinder abgebildet werden kann, während die bisherige Forschung zumeist nur die Perspektive der Eltern berücksichtig hat (vgl. die unter 2.1 angeführten Untersuchungen).

Indikatoren, die die Qualität der sozialen Beziehungen zwischen den Familienmitgliedern bezüglich schulischer Belange abbilden, sind: „Unterstützungsverhalten der Eltern schulische Belange betreffend“, welches für eine positive Bestärkung der Kinder durch die Eltern steht, ,Konflikte schulische Belange betreffend“, welche für eine Beeinträchtigung der schulbezogenen Beziehung zwischen Eltern und Kind stehen und „Erwartungen der Eltern schulische Belange betreffend“, im Sinne eines zu hohen Erwartungsdrucks seitens der Eltern.

- Unterstützungsverhalten der Eltern schulische Belange betreffend (Kindsicht) (faktorenanalytisch gebildet):

( 1 =stimmt nicht bis $4=$ stimmt) (Welle eins; Welle zwei)

Eltern fragen mich, wie es in der Schule war; Eltern freuen sich, wenn ich gute Noten bekomme; Eltern helfen mit, wenn es Schwierigkeiten wie z. B. Streit oder Ungerechtigkeiten in der Schule gibt

(drei Items: interne Konsistenz: Welle eins: 0,35; Welle zwei: 0,36.)

- Konflikte schulische Belange betreffend (Elternsicht):

( 1 =alltägliche Konflikte; $0=$ schulische Konflikte) (Welle zwei)

In welchem Bereich gibt es die meisten Unstimmigkeiten?

- Erwartungen der Eltern schulische Belange betreffend (Elternsicht) (faktorenanalytisch gebildet):

( $1=$ stimme überhaupt nicht $\mathrm{zu}$ bis $5=$ stimme voll und ganz $\mathrm{zu}$ ) (Welle eins; Welle zwei)

gute Noten, auch wenn es hart dafür arbeiten muss; Noten sind nicht so wichtig; schlechte Leistungen werden nicht toleriert; Wohlbefinden wichtiger als gute Noten (vier Items: interne Konsistenz: Welle eins: 0,55; Welle zwei: 0,50)

Indikatoren, die die Qualität der sozialen Beziehungen zwischen den Familienmitgliedern bezüglich alltäglicher Interaktion abbilden, sind: „Familienstimmung im Alltag“, „Ent- 
scheidungsfindung innerhalb der Familie im Alltag“ und „Respektvoller Umgang im Alltag" im Sinne eines harmonischen und gleichberechtigten familialen Miteinanders.

- Respektvoller Umgang im Alltag (Kindsicht):

( $1=$ nie bis $4=$ ja, immer) (Welle eins; Welle zwei)

Gefühl, von den Eltern ernst genommen zu werden

- Entscheidungsfindung innerhalb der Familie im Alltag (Kindsicht):

( 1 =gemeinsam; $0=$ Eltern entscheiden) (Welle eins; Welle zwei)

Wer entscheidet, wohin du in deiner Freizeit gehst?

- Familienstimmung im Alltag (Elternsicht) (faktorenanalytisch gebildet):

( 1 =trifft überhaupt nicht zu bis $5=$ trifft voll und ganz zu) (Welle zwei)

Zeit, einander zuzuhören; gegenseitige Unterstützung; kein Zusammengehörigkeitsgefühl; gemeinsame Aktivitäten; es kann über alles gesprochen werden; viele Konflikte

(sechs Items: interne Konsistenz: 0,70)

Integriert werden außerdem Familienstrukturvariablen, die die Quantität der sozialen Interaktion zwischen den Familienmitgliedern abbilden:

- Anzahl der Geschwister:

$(0=$ keine Geschwister bis $3=$ drei oder mehr Geschwister $)$

- und die Familiensituation:

( 1 =alleinerziehend; $0=$ nicht-alleinerziehend im Haushalt $)$.

Bezüglich der Güte der faktorenanalytisch gebildeten Indizes lässt sich sagen, dass „Familienstimmung im Alltag“ mit einer internen Konsistenz von 0,70 ausreichend hoch ist. „Erwartungen der Eltern schulische Belange betreffend“ weist in Anbetracht der Itemanzahl der Skala ebenfalls eine ausreichend hohe interne Konsistenz auf. Der Index „Unterstützungsverhalten der Eltern schulische Belange betreffen“ zeigt, auch aufgrund der niedrigen Itemanzahl, eine zu niedrige interne Konsistenz auf. Diese Itemstruktur (dies gilt auch für „Erwartungen der Eltern schulische Belange betreffend“) findet sich jedoch sowohl in Welle eins als auch in Welle zwei, so dass der Wert dennoch berücksichtigt wird. Alle drei Indizes weisen ebenso wie die mehrstufigen Items ,Respektvoller Umgang im Alltag“ und „Anzahl der Geschwister“ eine ausreichende hohe Streuung und keine Antworttendenzen auf. Das Kriterium der Normalverteilung ist hingegen nur für die Skala „Erwartungen der Eltern schulische Belange betreffend“ und das Item „Anzahl der Geschwister“ erfüllt. Die Indizes „Unterstützungsverhalten der Eltern schulische Belange betreffend“ und „Familienstimmung im Alltag“ sowie das Item „Respektvoller Umgang im Alltag“ weisen eine rechtsschiefe Verteilung auf. Dies stellt sich aufgrund ausreichender Zellbesetzung - in manchen Fällen durch das Zusammenfassen einzelner Fälle sichergestellt - allerdings unproblematisch dar. Die dichotomen Einzelitems „Konflikte schulische Belange betreffend“, „Entscheidungsfindung innerhalb der Familie im Alltag“ und „Familiensituation“ zeigen ebenfalls für jede Ausprägung ausreichend Fälle. 


\subsection{Methodisches Vorgehen}

Gemäß den oben angeführten Untersuchungen kann Bildungserfolg unterschiedlich operationalisiert werden. Innerhalb des deutschen Bildungssystems ist vor allem der Übergang in den Sekundarbereich ausschlaggebend für den späteren Bildungserfolg. Hierfür entscheidend ist vor allem das Erzielen eines bestimmten erreichten Notenlevels (niedriges Level 3,0-6,0; mittleres Level 2,67; hohes Level 1,0-2,3), welches in Bayern den institutionellen Regelungen geschuldet zumindest bedingt zu einem Übergang in einen niedrigen Bildungsgang (Hauptschule), einen mittleren Bildungsgang (Realschule) und hohen Bildungsgang (Gymnasium) nach der vierten Klasse berechtigt ${ }^{3}$ (vgl. Sekretariat der Ständigen Konferenz der Kultusminister der Länder in der Bundesrepublik Deutschland 2006).

Wichtig ist also letztlich nur, innerhalb welches Notenlevels der Notendurchschnitt der Kinder liegt und nicht, welchen Notendurchschnitt die Kinder an sich erzielen. Bildungserfolg als abhängige Variable wird dementsprechend - nicht als Notendurchschnitt und analog der drei Sekundarschulzweige - als dreistufiges Notenlevel (und nicht als binäre Variable) in die Analysen eingehen und Effekte unabhängiger Variablen werden mittels multinomialer logistischer Regressionen geschätzt. Da in Hessen die Wahl des Sekundarschulzweiges nach der vierten Klasse, trotz einer starken Orientierung an den Leistungen, letztlich Sache der Eltern ist (vgl. Sekretariat der Ständigen Konferenz der Kultusminister der Länder in der Bundesrepublik Deutschland 2006), beinhaltet jedes Modell von Anfang an die Bundeslandzugehörigkeit als Variable ( $1=$ Bayern; $0=$ Hessen), um diesen institutionellen Unterschied zu kontrollieren.

Das längsschnittliche Forschungsdesign bietet die Möglichkeit, die Noten aus der zeitlich vorgelagerten Welle als Kontrollvariable in das Modell aufzunehmen. Eine häufige Kritik an den Analysen (für einen Überblick vgl. Dika u. Singh 2002) bezüglich der Zusammenhänge familialer Beziehungen und dem Bildungserfolg ist der fehlende Beleg über die Richtung der Effekte. Querschnittliche Forschung lässt offen, inwiefern tatsächlich die Eltern-Kind-Beziehung auf den Bildungserfolg wirkt oder ob umgekehrt ein bestimmter Bildungserfolg der Kinder für die Art und Weise des Eltern-Kind-Kontaktes verantwortlich ist. Durch das Kontrollieren des Notenlevels zum ersten Messzeitpunkt (Notenlevel Mitte dritte Klasse: $3=3,0-6,0 ; 2=2,67 ; 1=1,0-2,3$ ) werden letztlich Effekte der unabhängigen Variablen auf eine Veränderung im Erreichen des Notenlevels geschätzt und so die kausale Richtung festgelegt.

Zur Kontrolle der Ressourcen der Familie wird gemäß der theoretischen Überlegungen nach Coleman der höchste familiale Bildungsabschluss $(1=$ mindestens Abitur; $0=$ anderer Abschluss) als Kontrollvariable zum Humankapital der Familie aufgenommen. Die finanziellen Ressourcen sollten aus theoretischer Perspektive über das Einkommen operationalisiert werden; da in diesem Fragebogenitem die Anzahl der fehlenden Werte aber besonders hoch ist ${ }^{4}$ werden die materiellen Möglichkeiten über die sozio-ökonomische Position der Familie berücksichtigt (höchster familialer ISEI-Wert). 


\section{Ergebnisse}

\subsection{Der Einfluss der Eltern-Kind-Beziehung auf schulischen Erfolg}

Zunächst werden Effekte sozialer Beziehungen zwischen den Akteuren auf das Notenniveau Anfang der vierten Klasse mittels multinomialer logistischer Regression geschätzt. Im zweiten Modell wird untersucht, ob unter Einbezug der ökonomischen Ressourcen und des Bildungshintergrundes des Elternhauses soziale Beziehungen einen eigenständigen, ressourcenunabhängigen Beitrag zum Bildungserwerb leisten können. Für die in Tab. 2 gezeigten Analysen stehen 1500 Fälle zur Verfügung. Die Differenz zur Ausgangsstichprobe ergibt sich sowohl durch fehlende Werte im Elterninterview, dem Schülerfragebogen und den kindbezogenen Einschätzbogen als auch durch Stichprobenausfälle von Welle eins zu Welle zwei.

Dargestellt werden odds-ratios: Werte zwischen null und eins stehen für eine geringere Chance, ein niedriges oder mittleres Notenlevel gegenüber einem hohen Notenlevel zu erreichen, während Werte größer als eins für eine höhere Chance stehen, ein niedriges oder mittleres Niveau zu erzielen. Dementsprechend haben Kinder aus alleinerziehenden Haushalten und Kinder mit vielen Geschwistern ein signifikant höheres Risiko auf das Erreichen eines niedrigeren Bildungsniveaus. Ein hohes wahrgenommenes Unterstützungsverhalten der Eltern bezüglich schulischer Belange reduziert die Gefahr, während Konflikte über schulische Angelegenheiten und zu hohe Erwartungen der Eltern das Risiko auf das Erreichen eines mittleren Notenlevels (im Gegensatz zu einem hohen Notenlevel) erhöhen. Der von den Kindern als respektvoll empfundene Umgang und eine berichtete gemeinsame Entscheidungsfindung erhöhen die Chancen auf das Erreichen eines hohen Notenniveaus ebenso wie eine von den Eltern berichtete positive Fami-

Tab. 2: Innerfamiliale Beziehungen und Notenniveau Anfang der vierten Klasse (W2)

\begin{tabular}{lcc}
\hline & Modell 1 & \\
\cline { 2 - 3 } & niedrig vs. hoch & mittel vs. hoch \\
\hline Bundesland & $1,08(0,20)$ & $1,19(0,18)$ \\
Notenniveau (W1) & $\mathbf{1 3 , 7 1}^{* * *}(0,13)$ & $\mathbf{3 , 0 1}^{* *}(0,12)$ \\
Anzahl der Geschwister (W2) & $\mathbf{1 , 3 0}^{*}(0,12)$ & $1,08(0,11)$ \\
alleinerziehend vs. nicht-alleinerziehend (W2) & $\mathbf{2 , 8 4}^{* *}(0,26)$ & $1,24(0,26)$ \\
Unterstützungsverhalten der Eltern (W2) & $0,88(0,08)$ & $\mathbf{0 , 8 5}^{*}(0,08)$ \\
Konflikte (W2) & $0,72(0,21)$ & $\mathbf{0 , 6 8}^{*}(0,20)$ \\
Erwartungen der Eltern (W2) & $1,04(0,04)$ & $\mathbf{1 , 0 8}^{*}(0,04)$ \\
Respektvoller Umgang (W2) & $\mathbf{0 , 7 9 *}(0,10)^{*}$ & $0,86(0,10)$ \\
Entscheidungsfindung (W2) & $\mathbf{0 , 6 2}^{*}(0,19)$ & $\mathbf{0 , 6 9}(0,17)$ \\
Familienstimmung (W2) & $\mathbf{0 , 8 4}^{+}(0,10)$ & $0,91(0,09)$ \\
Nagelkerkes $R^{2}: 0,584 ; n=1500$ & &
\end{tabular}

${ }^{*}$ Effekt signifikant bei $\mathrm{p}<0,05 ;{ }^{* *}$ Effekt signifikant bei $\mathrm{p}<0,01$; ${ }^{+}$Effekt signifikant bei $\mathrm{p}<0,10$ Ergebnisse multinomialer logistischer Regression (odds ratios) (Standardfehler in Klammern) 
Tab. 3: Innerfamiliale Beziehungen und Notenniveau unter Kontrolle Ressourcen (W2)

\begin{tabular}{lcc}
\hline & Modell 2 & \\
\cline { 2 - 3 } & niedrig vs. hoch & mittel vs. hoch \\
\hline Bundesland & $1,09(0,20)$ & $1,21(0,18)$ \\
Notenniveau & $\mathbf{1 2 , 2 4}^{* *}(0,13)$ & $\mathbf{2 , 8 1}^{* *}(0,12)$ \\
Bildungshintergrund & $\mathbf{0 , 5 0}^{\text {*** }}(0,23)$ & $\mathbf{0 , 6 9}^{+}(0,20)$ \\
sozio-ökonomische Position & $\mathbf{0 , 9 8}^{\text {*** }}(0,01)$ & $0,99(0,01)$ \\
Anzahl der Geschwister (W2) & $\mathbf{1 , 2 7}^{*}(0,12)$ & $1,07(0,11)$ \\
alleinerziehend vs. nicht-alleinerziehend (W2) & $\mathbf{2 , 6 5}^{* *}(0,26)$ & $1,19(0,26)$ \\
Unterstützungsverhalten der Eltern (W2) & $0,89(0,09)$ & $\mathbf{0 , 8 5}^{*}(0,08)$ \\
Konflikte (W2) & $0,73(0,22)$ & $\mathbf{0 , 6 9}^{+}(0,20)$ \\
Erwartungen der Eltern (W2) & $1,01(0,04)$ & $\mathbf{1 , 0 6}^{+}(0,04)$ \\
Respektvoller Umgang (W2) & $\mathbf{0 , 7 9}^{*}(0,11)$ & $0,87(0,10)$ \\
Entscheidungsfindung (W2) & $\mathbf{0 , 6 4}^{*}(0,19)$ & $\mathbf{0 , 7 1}^{*}(0,17)$ \\
Familienstimmung (W2) & $\mathbf{0 , 8 2}^{*}(0,10)$ & $0,89(0,09)$ \\
Nagelkerkes $R^{2}: 0,597 ; n=1500$ & & \\
\hline
\end{tabular}

${ }^{*}$ Effekt signifikant bei $\mathrm{p}<0,05 ;{ }^{* *}$ Effekt signifikant bei $\mathrm{p}<0,01$; ${ }^{+}$Effekt signifikant bei $\mathrm{p}<0,10$

Ergebnisse multinomialer logistischer Regression (odds ratios) (Standardfehler in Klammern)

lienstimmung. Die Bundeslandzugehörigkeit spielt beim Erreichen von einem der drei Notenniveaus keine Rolle.

Nach Einbezug des Humankapitals der Eltern und der ökonomischen Ressourcen des Haushalts, abgebildet in Tab. 3, kann festgestellt werden, dass die Eltern-Kind-Beziehung tatsächlich einen signifikant eigenständigen Effekt auf Bildungserfolg hat und damit nicht an die dem Haushalt zur Verfügung stehenden Ressourcen gebunden ist. Hervorzuheben ist dabei insbesondere auch, dass die Effektstärken sozialer Beziehungen mit denen von Humankapital und ökonomischen Ressourcen vergleichbar sind und deshalb keine untergeordnete Rolle beim Erreichen von übergangsrelevanten Notenniveaus einnehmen.

Außerdem bestätigt sich, dass Indikatoren einer schulbezogenen Interaktion zwischen Eltern und Kind eher zwischen einem Notenniveau, das für den Übergang auf das Gymnasium berechtigt, und einem mittleren Notenniveau, das für den Übergang auf die Realschule berechtigt, trennen, während der nicht-schulbezogene Umgang der Eltern mit den Kindern (quantitativ und qualitativ) eher zwischen einem hohen und einem niedrigen Notenniveau trennt. Dabei zeigen sich sowohl die elterliche Wahrnehmung der Beziehung als auch die von den Kindern berichtete Interaktion als bedeutsame Prädiktoren schulischen Erfolges.

\subsection{Veränderungen der Eltern-Kind-Beziehung und schulischer Erfolg}

Im dritten Modell soll untersucht werden, inwiefern sich Veränderungen in der ElternKind-Interaktion über den Zeitraum eines halben Jahres auf das Erzielen eines bestimmten Notenlevels niederschlagen. Einbezogen werden können dabei Veränderungen für 
die Indikatoren „Erwartungen der Eltern schulische Belange betreffend“, „Respektvoller Umgang im Alltag“, „Unterstützungsverhalten der Eltern schulische Belange betreffend“ und „Entscheidungsfindung innerhalb der Familie im Alltag“. ${ }^{5}$ Mittels t-Tests kann vorab gezeigt werden, dass sich die gültigen Mittelwerte des Indikators „Respektvoller Umgang im Alltag“ über die beiden Wellen signifikant $(\mathrm{p}<0,01)$ unterscheiden. Die Mittelwerte der Indikatoren „Unterstützungsverhalten der Eltern schulische Belange betreffend“, „Entscheidungsfindung im Alltag“ und „Erwartungen der Eltern schulische Belange betreffend" zeigen sich dagegen über beide Wellen als statistisch nicht signifikant unterschiedlich. Dementsprechend verändert sich das Niveau der Eltern-Kind-Interaktion nur zum Teil in eine bestimmte Richtung, je näher der Übergang rückt: Der Umgang im Alltag wird von den Kindern als respektvoller berichtet und verändert sich demnach positiv. Für die anderen Interaktionen kann keine eindeutige Veränderung im Sinne einer allgemeinen, gruppenbezogenen Verbesserung oder Verschlechterung gezeigt werden.

Im nächsten Schritt wird unter Einbezug von Differenzscores (Werte aus Welle zwei werden von Werten in Welle eins subtrahiert) analysiert, inwiefern Veränderungen des familialen Miteinanders für die einzelnen Kinder ${ }^{6}$ Effekte auf das Notenniveau haben. Dabei ist bei der Interpretation der Werte zu beachten, dass mit steigendem Differenzwert die Beziehung zwischen Eltern und Kind schlechter wurde. Kontrolliert werden das Ausgangsniveau der sozialen Beziehungen, der familialen Ressourcen und die Bundeslandzugehörigkeit. Zur Beantwortung der Fragestellung stehen 672 Fälle zur Verfügung. Der Unterschied hinsichtlich der Fallzahlen ergibt sich, neben Stichprobenausfällen und fehlenden Angaben, designbedingt. In Welle eins wurden zwei Versionen des Schülerfragebogens vorgelegt, so dass von vornherein nur für die Hälfte der Kinder Antworten zu den relevanten Items vorliegen.

Tabelle 4 zeigt, dass die beobachtete Verbesserung im Unterstützungsverhalten der Eltern bezüglich schulischer Belange sich signifikant positiv auf das Erreichen höherer Notenlevel auswirkt. Kinder, die in der vierten Klasse über ein schlechteres Unterstützungsverhalten der Eltern als in der dritten Klasse berichten, haben ein höheres Risiko auf das Erreichen von niedrigen und mittleren Notenleveln als auf das Erreichen eines hohen Notenlevels. Das Ausgangsniveau dieser Interaktion wird hingegen nicht signifikant, so dass also nicht bedeutend ist, von welchem Ausgangspunkt die Beziehung sich verändert, sondern dass es eine Veränderung gibt. Eine Veränderung der Erwartungen der Eltern hingegen zeigt keinen Einfluss auf eine Leistungsänderung. Genauso zeigen auch Veränderungen hinsichtlich des alltäglichen Umgangs der Eltern mit den Kindern keine Effekte auf das Erreichen höherer Notenlevel. Ebenfalls unbedeutend ist für diese Beziehungen das Ausgangsniveau der sozialen Beziehung Mitte der dritten Klasse. In diesen Fällen ist dementsprechend das Niveau des sozialen Umgangs Anfang der vierten Klasse entscheidend für Veränderungen in den schulischen Leistungen und nicht die Veränderung in der sozialen Beziehung oder die frühe soziale Beziehung.

Ebenfalls signifikant unbedeutend ist Bildung oder die sozio-ökonomische Position des Elternhauses bzw. das Bundesland; für eine Veränderung im erreichten Notenlevel über diesen Zeitraum ist es demnach nicht wichtig, welche sozialen Merkmale die Eltern haben, sondern wie sich die Eltern bezüglich schulischer Belange verhalten. 
Tab. 4: Innerfamiliale Beziehungen und Notenniveau unter Einbezug Veränderungen (W2)

\begin{tabular}{lcc}
\hline & Modell 3 \\
\cline { 2 - 3 } & niedrig vs. hoch & mittel vs. hoch \\
\hline Bundesland & $1,10(0,30)$ & $1,12(0,27)$ \\
Notenniveau & $\mathbf{1 5 , 9 6}^{* *}(0,21)$ & $\mathbf{3 , 3 3}^{* *}(0,21)$ \\
Bildungshintergrund & $0,78(0,37)$ & $0,72(0,31)$ \\
sozio-ökonomische Position & $0,98(0,01)$ & $1,00(0,01)$ \\
Erwartungen der Eltern (W1) & $1,00(0,07)$ & $1,02(0,07)$ \\
Respektvoller Umgang (W1) & $0,72(0,20)$ & $0,83(0,19)$ \\
Unterstützungsverhalten der Eltern (W1) & $0,85(0,17)$ & $0,89(0,16)$ \\
Entscheidungsfindung (W1) & $0,71(0,40)$ & $0,77(0,34)$ \\
Veränderung Erwartungen der Eltern (W1-W2) & $1,06(0,07)$ & $0,95(0,06)$ \\
Veränderung respektvoller Umgang (W1-W2) & $1,16(0,17)$ & $0,91(0,17)$ \\
Veränderungen Verhalten der Eltern (W1-W2) & $\mathbf{1 , 4 2}^{*}(0,14)$ & $\mathbf{1 , 4 3}(0,12)$ \\
Veränderung Entscheidungsfindung (W1-W2) & $1,50(0,30)$ & $1,00(0,26)$ \\
Nagelkerkes $R^{2}: 0,608 ; n=672$ & & \\
\hline
\end{tabular}

${ }^{*}$ Effekt signifikant bei $\mathrm{p}<0,05 ;{ }^{* *}$ Effekt signifikant bei $\mathrm{p}<0,01$; ${ }^{+}$Effekt signifikant bei $\mathrm{p}<0,10$

Ergebnisse multinomialer logistischer Regression (odds ratios) (Standardfehler in Klammern)

\section{Zusammenfassung und Ausblick}

Die vorliegenden Analysen befassen sich mit der Relevanz innerfamilialer Beziehungen für den Bildungserfolg eines Kindes während der Grundschulzeit - gemessen am Erreichen eines bestimmten Notenlevels. Einflüsse sozialer Beziehungen auf Bildungserfolg konnten in zahlreichen Studien belegt werden (vgl. Abschn. 2.2). Repliziert werden konnten diese Ergebnisse für eine frühe Phase in der Bildungskarriere eines Kindes. Die Analysen zeigen, dass sowohl die Quantität als auch die Qualität der Eltern-Kind-Beziehung signifikant positive Effekte auf das Erreichen eines hohen Notenniveaus und damit auf Bildungserfolg aufweisen. Die untersuchten Merkmale der Struktur und des Rahmens der Beziehung zwischen Eltern und Kind sind also ebenso wichtig wie die tatsächliche Art und Weise des Umgangs. Das heißt, dass die Möglichkeit zu interagieren genauso relevant ist wie das, was aus dieser Möglichkeit gemacht wird. Wenngleich vorliegende Indikatoren nur einen Ausschnitt des familialen Miteinanders abbilden können, wird in den Analysen dennoch deutlich, dass Indikatoren einer schulbezogenen Interaktion zwischen Eltern und Kind genauso wirken wie Indikatoren eines nicht-schulbezogenen Eltern-Kind-Umgangs. Sozialkapital, als für den Bildungserfolg bedeutende Ressource, liegt also generell in der Beziehung zwischen den Familienmitgliedern und eben nicht ausschließlich in Interaktionen mit explizitem schulischen Bezug. Dies spricht - ganz im Sinne Colemans - für die grundsätzliche Bedeutung einer engen Eltern-Kind-Beziehung für den Erwerb von Humankapital, nicht nur in humankapitalreichen Haushalten.

Unterstützt wird dies weiterhin durch einen ähnlich starken Zusammenhang zwischen diesen Prozessen und Strukturen des familialen Miteinanders als familiale Ressource und den Schulleistungen wie der Zusammenhang zwischen dem familialen Humankapital oder den ökonomischen Ressourcen und den Noten. Innerfamiliale Beziehungen leisten 
demnach einen eigenständigen und bedeutsamen Beitrag zur Aufklärung des Zusammenhangs zwischen familialen Ressourcen und Bildungserfolg. Colemans Hypothese, dass Sozialkapital die dritte bedeutende Ressource des familialen Haushaltes sei und unabhängig anderer Ressourcen nachgewiesen werden könne, kann damit mit diesen Daten gezeigt werden.

Dargestellt werden kann mit den vorliegenden Daten außerdem, dass die schulbezogene Interaktion zwischen Eltern und Kind eher eine Differenzierung zwischen den Übergängen auf die höheren Sekundarschulzweige ermöglicht, während der alltägliche Umgang innerhalb der Familie zwischen einem hohen Notenlevel und einem niedrigen Notenlevel separiert. Unter Berücksichtigung der Einschränkungen durch die begrenzte Anzahl an Indikatoren lässt sich als Erweiterung zum bisherigen Forschungsstand für den frühen Bildungsverlauf zeigen, dass Interaktion über schulische Angelegenheiten ausschlaggebend für Bildungserfolg auf einem höheren Niveau sein kann; das alltägliche Miteinander hingegen eher darüber entscheidet, ob man ein hohes Niveau erreicht oder nicht.

Die theoretischen Annahmen Colemans sowie Forschungsergebnisse, die diese Annahmen untersuchten, lassen sich dementsprechend - Bezug nehmend auf die eingangs formulierten Forschungsfragen - auch zu einem frühen Zeitpunkt der Bildungslaufbahn bestätigen und ergänzen. Die längsschnittliche Perspektive bietet desgleichen eine Erweiterung des bisherigen Forschungsstandes und lässt eine Interpretation der Wirkrichtung des Zusammenhangs zwischen den innerfamilialen Beziehungen und Bildungserfolg zu. Die sozialen Beziehungen innerhalb der Familie sind unter dieser Perspektive nicht Ergebnis einer bestimmten schulischen Leistung des Kindes, sondern nehmen Einfluss auf die Bildungsergebnisse. Ein Einfluss von schulischen Leistungen auf die Eltern-KindBeziehung wird dadurch nicht generell ausgeschlossen; lediglich findet für die Interpretation eine Festlegung der Richtung der Effekte statt.

Unter längsschnittlicher Perspektive kann außerdem die Frage beantwortet werden, ob sich innerfamiliale Beziehungen generell ändern, je näher der entscheidende Übergang rückt. Mit der Einschränkung, dass der Zeitraum eines halben Jahres betracht wird, zeigt sich, dass sich das Niveau der Eltern-Kind-Interaktion nur für den respektvollen Umgang miteinander signifikant ändert; d.h., es ist eine positive Veränderung der Qualität in eine bestimmte Richtung zu verzeichnen, je näher der Übergang rückt. Für die Interaktionen bezüglich schulischer Belange, den Erwartungen der Eltern und die familiale Entscheidungsfindung ergeben sich keine Veränderungen in eine bestimmte Richtung; d.h., das familiale Miteinander erfährt sowohl positive als auch negative Veränderungen. Dennoch verändert sich das familiale Miteinander für eine signifikant große Anzahl von Kindern. Diese Veränderungen in den sozialen Beziehungen erweisen sich dann für den einzelnen als bedeutsam auf Veränderungen im Notenniveau, wenn die Beziehung vom Kind wahrgenommen wird und darüber hinaus sich elterliches Verhalten in Bezug auf schulische Belange widerspiegelt. Aus dieser Perspektive ist sowohl die Sicht des Akteurs - welche in querschnittlicher Betrachtung keinen Unterschied machte - wichtig als auch der konkrete schulische Bezug der Eltern-Kind-Beziehung im Zeitverlauf. Diese Veränderung der Eltern-Kind-Beziehung zeigt sich für die Veränderung im erreichten Notenlevel bedeutsamer als die soziale Herkunft der Kinder. Dieses unterstreicht erneut die Relevanz von Sozialkapital für den Bildungserfolg. Allerdings muss erneut berücksichtigt werden, dass 
lediglich Veränderungen über den Zeitraum eines halben Jahres untersucht werden. Veränderungen über mehrere Messzeitpunkte können näheren Aufschluss bringen.

Zukünftige Analysen sollen die Bedeutung innerfamilialer Beziehungen für Bildungserfolge in der Sekundarschulzeit näher beleuchten. Dabei können der tatsächlich vollzogene Übergang oder die Wechsel innerhalb der verschiedenen Schulzweige betrachtet werden, aber auch Unterschiede in der Relevanz innerfamilialer Beziehungen zwischen den Schultypen ins Auge gefasst werden. Die längsschnittliche Perspektive sollte auch für diesen Zeitpunkt der Bildungskarriere sowohl aus methodischer als auch aus inhaltlicher Perspektive beibehalten werden.

Open Access Dieser Artikel unterliegt den Bedingungen der Creative Commons Attribution Noncommercial License. Dadurch sind die nichtkommerzielle Nutzung, Verteilung und Reproduktion erlaubt, sofern der/die Originalautor/en und die Quelle angegeben sind.

\section{Anmerkungen}

1 Die vorliegende Arbeit ist entstanden im Rahmen der von der Deutschen Forschungsgemeinschaft geförderten interdisziplinären Forschergruppe BiKS im Teilprojekt 1 (Leitung: Prof. Dr. Cordula Artelt; Prof. Dr. Hans-Peter Blossfeld; Prof. Dr. Gabriele Faust; Prof. Dr. HansGünther Roßbach; Prof. Dr. Sabine Weinert; RO-820-11). Wir danken den an der Studie teilnehmenden Kindern, Lehrerinnen und Lehrern und Eltern für ihre Teilnahme und allen im Rahmen der Datenerhebungen eingesetzten Studierenden für ihre engagierte Mitarbeit.

2 Günstiger wäre es, Indikatoren zu nutzen, die Veränderungen über den Zeitraum eines Jahres abdecken. Allerdings ist die Fülle an Indikatoren nur in den verwendeten Wellen eins und zwei vorzufinden. Das Notenniveau unterscheidet sich aber auch über den Zeitraum eines halben Jahres signifikant $(\mathrm{p}<0,01)$.

3 Die Eignung für das Gymnasium liegt vor, wenn die Gesamtdurchschnittsnote mind. 2,33 beträgt; beträgt der Notendurchschnitt aus Deutsch und Mathe nicht mind. 2,0, wird eine bedingte Eignung festgestellt. Die Eignung für die Realschule liegt vor, wenn die Gesamtdurchschnittsnote mind. 2,33 beträgt; beträgt sie 2,66, wird eine bedingte Eignung festgestellt. Schülerinnen und Schüler, die nicht geeignet sind, deren Eltern aber den Übertritt wünschen, nehmen am Probeunterricht teil.

4 Bei knapp 93\% (2,225) Teilnahmebereitschaft am Elterninterview in der ersten Welle, machten nur knapp 70\% (1,552) der Interviewten Angaben zu ihrer Einkommenssituation, während der ISEI für fast 95\% $(2,073)$ der Interviewten gebildet werden konnte.

5 „Familienstimmung im Alltag“ und „Konflikte schulische Belange betreffend“ finden sich nur in Welle zwei. Die Familienstrukturvariablen zeigen keine signifikant ausreichende Anzahl an Veränderungen.

6 Analysen mittels Messwiederholungsdesigns bestätigen die Ergebnisse der Mittelwertvergleiche und zeigen darüber hinaus ausreichend Varianz zwischen den Kindern, weswegen alle Indikatoren der sozialen Interaktion in die Analysen aufgenommen werden. 


\section{Literatur}

Baumert, J., Klieme, E., Neubrand, M., Prenzel, M., Schiefele, U., Schneider, W., Stanat, P., Tillmann, K.-J., \& Weiß, M. (Hrsg.). (2001). PISA 2000: Basiskompetenzen von Schülerinnen und Schülern im internationalen Vergleich. Opladen: Leske + Budrich.

Becker, R. (2000). Klassenlage und Bildungsentscheidungen. Eine empirische Anwendung der WertErwartungstheorie. Kölner Zeitschrift für Soziologie und Sozialpsychologie, 52, 450-474.

Becker, R. (2004). Soziale Ungleichheit von Bildungschancen. In R. Becker \& W. Lauterbach (Hrsg.), Bildung als Privileg? Erklärungen und Befunde zu den Ursachen der Bildungsungleichheit (3. Aufl., S. 161-195). Wiesbaden: VS Verlag für Sozialwissenschaften.

Below, S. von (2002). Bildungssysteme und soziale Ungleichheit. Das Beispiel der neuen Bundesländer. Opladen: Leske + Budrich.

Bos, W., Voss, A., Lankes, E.-M., Schwippert, K., Thiel, O., \& Valtin, R. (2004). Schullaufbahnempfehlungen von Lehrkräften für Kinder am Ende der vierten Jahrgangsstufe. In W. Bos, E.-M. Lankes, M. Prenzel, K. Schwippert, R. Valtin, \& G. Walther (Hrsg.), IGLU. Einige Länder der Bundesrepublik Deutschland im nationalen und internationalen Vergleich (S. 191-228). Münster: Waxmann.

Blossfeld, H.-P. (1988). Sensible Phasen im Bildungsverlauf. Eine Längsschnittanalyse über die Prägung von Bildungskarrieren durch gesellschaftlichen Wandel. Zeitschrift für Pädagogik, 34, 45-64.

Blossfeld, H.-P. (1993). Changes in educational opportunities in the Federal Republic of Germany. A longitudinal study of cohorts born between 1916 and 1965. In Y. Shavit \& H.-P. Blossfeld (Hrsg.), Persistent inequality. Changing educational attainment in thirteen countries (pp. 51-74). Boulder: Westview Press.

Bourdieu, P. (1983). Ökonomisches Kapital, kulturelles Kapital, soziales Kapital. In R. Kreckel (Hrsg.), Soziale Ungleichheiten (Soziale Welt: Sonderbd. 2., S. 183-198). Göttingen: Schwartz.

Büchel, F., \& Wagner, G. (1996). Soziale Differenzen der Bildungschancen in Westdeutschland Unter besonderer Berücksichtigung von Zuwandererkindern. In W. Zapf, J. Schupp, \& R. Habich (Hrsg.), Lebenslagen im Wandel: Sozialberichterstattung im Längsschnitt (S. 80-96). Frankfurt a.M.: Campus.

Büchner, P., \& Koch, K. (2001). Von der Grundschule in die Sekundarstufe. Der Übergang aus Kinder- und Elternsicht (Bd. 1). Opladen: Leske + Budrich.

Coleman, J. S. (1988). Social capital in the creation of human capital. American Journal of Sociology, 94, 95-120.

Coleman, J. S. (1990). Foundations of social theory. Cambridge: Belknap Press of Harvard University Press.

Dika, S. L., \& Singh, K. (2002). Application of social capital in educational literature. A critical synthesis. Review of Educational Research, 72, 31-60.

Ditton, H., Krüsken, J., \& Schauenberg, M. (2005). Bildungsungleichheit - der Beitrag von Familie und Schule. Zeitschrift für Erziehungswissenschaft, 8, 285-305.

Dohle, K. \& Wessel, A. (1997). Soziales Kapital von Grundschülern und ihr Schulerfolg in der Oberschule. Unveröff. Vortragsdokument der AEPF-Tagung vom 1.-4.10.1997.

Ehmke, T., Siegle, T., \& Hohensee, F. (2005). Soziale Herkunft im Ländervergleich. In M. Prenzel, J. Baumert, W. Blum, R. Lehmann, D. Leutner, M. Neubrand, R. Pekrun, H.-G. Rolff, J. Rost, \& U. Schiefele (Hrsg.), PISA 2003 - Der zweite Vergleich der Länder in Deutschland. Was wissen und was können Jugendliche? (S. 233-264). Münster: Waxmann.

Ganzeboom, H., De Graaf, P. M., \& Treiman, D. J. (1992). A standard international socio-economic index of occupational status. Social Science Research, 21(1), 1-56.

Henz, U., \& Maas, I. (1995). Chancengleichheit durch die Bildungsexpansion? Kölner Zeitschrift für Soziologie und Sozialpsychologie, 47, 605-633. 
Hillmert, S., \& Jacob, M. (2005). Zweite Chance im Schulsystem? Zur sozialen Selektivität bei „späteren“ Bildungsentscheidungen. In P. A. Berger \& H. Kahlert (Hrsg.), Institutionalisierte Ungleichheiten? Wie das Bildungswesen Chancen blockiert (S. 155-176). Weinheim: Juventa.

Ho Sui-Cho, E., \& Willms, J. D. (1996). Effects of parental involvement on eight-grade achievement. Sociology of Education, 69, 126-141.

Israel, G. D., \& Beaulieu, L. J. (2002). The influence of social capital on test scores: How much do families, schools and communities matter? http://aaae.okstate.edu/regions/southern/02.israel. beaulieu.paper.pdf. Zugegriffen 29. Okt. 2008.

Israel, G. D., Beaulieu, L. J., \& Hartless, G. (2001). The influence of family and community social capital on educational achievement. Rural Sociology, 66, 43-68.

Jungbauer-Gans, M. (2004). Einfluss des sozialen und kulturellen Kapitals auf die Lesekompetenz (Ein Vergleich der PISA 2000 Daten aus Deutschland, Frankreich und der Schweiz). Zeitschrift für Soziologie, 33, 375-397.

Kristen, C. (2002). Hauptschule, Realschule oder Gymnasium? Ethnische Unterschiede am ersten Bildungsübergang. Kölner Zeitschrift für Soziologie und Sozialpsychologie, 54, 534-552.

Kurz, K., Kratzmann, J., \& Maurice, J. von (2007) Die BiKS-Studie. Methodenbericht zur Stichprobenziehung. PsyDok [Online], 2007/990. URN: urn:nbn:de. bsz:291-psydok-9901; URL: http://psydok.sulb.uni-saarland.de/volltexte/2007/990/. Zugegriffen 10. Feb. 2009.

Maurice, J. von, Artelt, C., Blossfeld, H.-P., Faust, G., Roßbach, H.-G., \& Weinert, S. (2007). Bildungsprozesse, Kompetenzentwicklung und Formation von Selektions-entscheidungen im Vor- und Grundschulalter. Überblick über die Erhebungen in den Längsschnitten BiKS-3-8 und BiKS-8-12 in den ersten beiden Projektjahren. PsyDok [Online], 2007/1008. URN: urn: nbn:de:bsz:291-psydok-10089; URL: http://psydok.sulb.uni-saarland.de/volltexte/2007/1008/. Zugegriffen 10. Feb. 2009.

Müller, W., \& Haun, D. (1994). Bildungsungleichheit im sozialen Wandel. Kölner Zeitschrift für Soziologie und Sozialpsychologie, 46, 1-42.

Pohlmann, S. (2008). Der Übergang von der Primar- in die Sekundarstufe aus der Sicht bayerischer und hessischer Lehrer. Diskurs Kindheits- und Jugendforschung, 3, 123-140.

Prenzel, M., Baumert, J., Blum, W., Lehmann, R., Leutner, D., Neubrand, M., Pekrun, R., Rolff, H.-G., Rost, J., \& Schiefele, U. (Hrsg.). (2004). PISA 2003. Der Bildungsstand der Jugendlichen in Deutschland. Ergebnisse des zweiten internationalen Vergleichs. Münster: Waxmann.

Schimpl-Neimanns, B. (2000). Soziale Herkunft und Bildungsbeteiligung: Empirische Analysen zu herkunftsspezifischen Bildungsungleichheiten zwischen 1950 und 1989. Kölner Zeitschrift für Soziologie und Sozialpsychologie, 52, 636-669.

Sekretariat der Ständigen Konferenz der Kultusminister der Länder in der Bundesrepublik Deutschland (2006). Übergänge von der Grundschule in Schulen des Sekundarbereichs I. Kultusministerkonferenz online. www.kmk.org/doc/publ/ueberg.pdf. Zugegriffen 10. Dez. 2008.

Smith, M. H., Beaulieu, L. J., \& Israel, G. (1992). Effects of human capital and social capital on dropping out of high school in the south. Journal of Research in Rural Education, 8, 75-88.

Smith, M. H., Beaulieu, L. J., \& Seraphine, A. (1995). Social capital, place of residence and college attendance. Rural Sociology, 60, 363-381.

Stecher, L. (2001). Die Wirkung sozialer Beziehungen. Empirische Ergebnisse zur Bedeutung sozialen Kapitals für die Entwicklung von Kindern und Jugendlichen. Weinheim: Juventa.

Teachman, J. D., Paasch, K., \& Carver, K. (1996). Social capital and dropping out of school early. Journal of Marriage and the Family, 58, 773-783. 